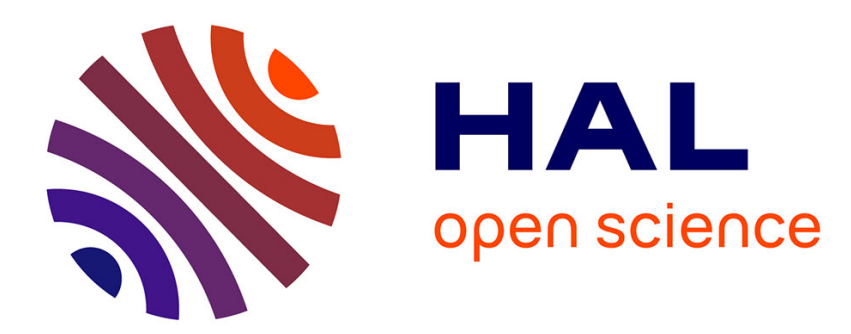

\title{
AB INITIO FORCE CONSTANTS OF GERMANIUM
}

\author{
K. Kunc, Roland Martin
}

\section{To cite this version:}

K. Kunc, Roland Martin. AB INITIO FORCE CONSTANTS OF GERMANIUM. Journal de Physique Colloques, 1981, 42 (C6), pp.C6-649-C6-651. 10.1051/jphyscol:19816189 . jpa-00221272

\section{HAL Id: jpa-00221272 https://hal.science/jpa-00221272}

Submitted on 1 Jan 1981

HAL is a multi-disciplinary open access archive for the deposit and dissemination of scientific research documents, whether they are published or not. The documents may come from teaching and research institutions in France or abroad, or from public or private research centers.
L'archive ouverte pluridisciplinaire HAL, est destinée au dépôt et à la diffusion de documents scientifiques de niveau recherche, publiés ou non, émanant des établissements d'enseignement et de recherche français ou étrangers, des laboratoires publics ou privés. 
K. Kunc and R.M. Martin

CNRS and Université P. et M. Curie, Tour 13-22, 4 p2. Jussieu, 75230 Paris cedex 05, France

Xerox Research Center, 3333 Coyote Hill Road, Palo Alto, Califomia 94304, U.S.A.

\begin{abstract}
Using the local density-functional formalism, phonon frequencies can be $\overline{\text { predicted }} a b$ initio by comparing the energy of the equilibrium configuration with the one having the atoms displaced in a pattern corresponding to a "frozen" phonon. Here we show that by using less symmetric displacement patterns and by applying the Hellman-Feynman theorem to the self-consistent electronic charge densities, one can obtain all force constants determining the entire branches of phonon spectra corresponding to a selected direction of propagation. Our method is illustrated on the example of $r-x$ branches of phonon spectra in Ge.
\end{abstract}

The local density-functional formalism [1] which was recently applied to $a b$ initio evaluation of phonon frequencies in $\mathrm{Si}$ [2] and GaAs [3] showed an astounding accuracy in predicting total energies of semiconductors: Not only the phonon energies (given in the "frozen phonon" approach as a difference between the energies of two configurations of atoms) but also Gruneisen parameters were accurately determined. The (unknown) eigenvectors of the $\operatorname{TO}(X)$ and $T A(X)$ modes in GaAs were established and their comparison with (extremely varied) predictions of different phenomenological models made it possible to judge the physical realism of the latter. The method also proved to be useful for determination of effective charges [3], for studying anharmonicity, and for predicting [3] a phase transition of GaAs to orthorhombic structure, not yet known experimentally. In this paper we show that it is possible to go still further by applying the Hellman-Feynman theorem to the same functional of the electronic density; by proceeding in terms of forces on all the ions we can obtain much more complete picture of interactions in solid than from the enrgies alone - providing that we study less symmetric displacement patterns than those of isolated phonon eigenmodes.

Let us consider phonon propagating in Ge along the [100] direction; then at any $k=(k, 0,0)$ the $(100)$ atomic planes vibrate as rigid units with respect to each other and oscillations of the set of (100) planes can be assimilated to vibrations of linear chain. The interplanar force constants needed for writing down the dynamical matrix of such a chain can be obtained in geometry shown in Fig. 1: The elementary unit cell of Ge was quadrupled along the [100] direction and one of the atoms was displaced from the equilibriun position by $u=-0.01 a$ in the direction [011]. With this periodic supercell and using the Berkeley ionic pseudopotential [4] the self-consistent charge densities were evaluated in very much te same way as e.g. in Ref. 5 . 


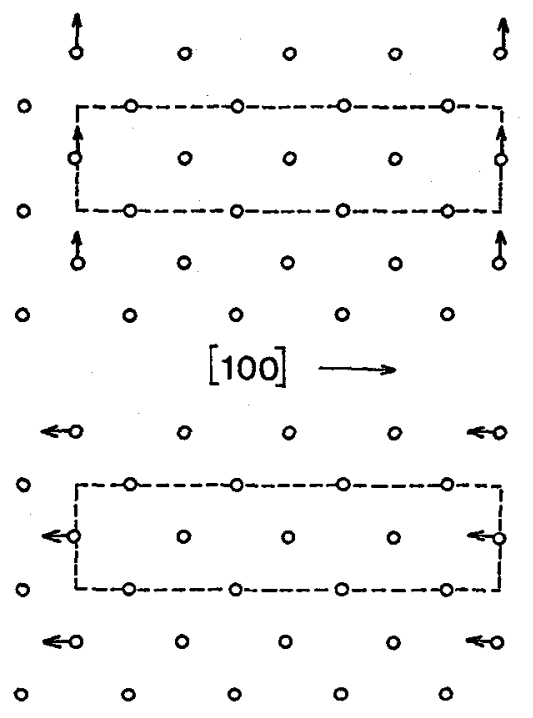

Fig. 1: Description of all phonons propagating in the [100] direction requires self-consistent charge densities determined on periodic supercells which can be constructed by repeating the elementary unit cell along the [100] direction. Present calculations were performed on this quadrupled cell containing 8 atoms of basis.

(a) Displacement pattern allowing to establish the forces determining the transverse vibrations.

(b) Pattern leading to longitudinal force constants.

(b)

Applying the Hellman-Feynman theorem we obtained the forces acting on all the neighboring planes extending up to the fourth neighbors. The $2 \times 2$ dynamical matrix of the "linear chain" is easily constructed and by diagonalizing it for different wavevectors $k$ we obtain the transverse acoustic and optical branches given in Fig. 2 (solid lines). Starting from the longitudinal configuration of Fig. Ib one obtains a different set of force parameters - which determine the longitudinal branches given in Fig. 2, as well.

Internal consistency of our calculations can be judged from the fact that the two sets of force parameters, which were provided by two independent and completely different calculations, give the $\mathrm{LO}$ and TO branches converging to the same $\mathrm{LTO}(\boldsymbol{r})$ frequency degenerate to within $1.1 \%$. The energy of this mode agrees with our prediction using the "frozen phonon" approach (i.e. proceeding via the total energies) to within $0.2 \%$ - which illustrates the consistency of both methods.

The main reason for an imperfect agreement between the experiment and theory is in the pseudopotential of $\mathrm{Ge}$ which was shown in [5] to predict the equilibrium lattice constant of $\mathrm{Ge}$ by $8 \%$ too short; this gives a spurious contribution to all forces. Correcting for this term we obtain a second set of dispersion curves plotted in Fig. 2 (broken lines). The agreement with experiment is still imperfect - for which four mechanisms can be suspected to be reponsible: (1) merely computational problems: sampling ( 8 special points were used in the transverse and 4 in the longitudinal case), plane wave expansions $(\approx 580$ waves), self-consistency (to within less than $2 \times 10^{-5} R y$.in the components of potential with small $\mathrm{G}$-vectors and less than $0.1 \%$ in all other ones); (2) anharmonicity; (3) method of correcting the inadequacy of pseudopotential and (4) limited range of force constants considered. 


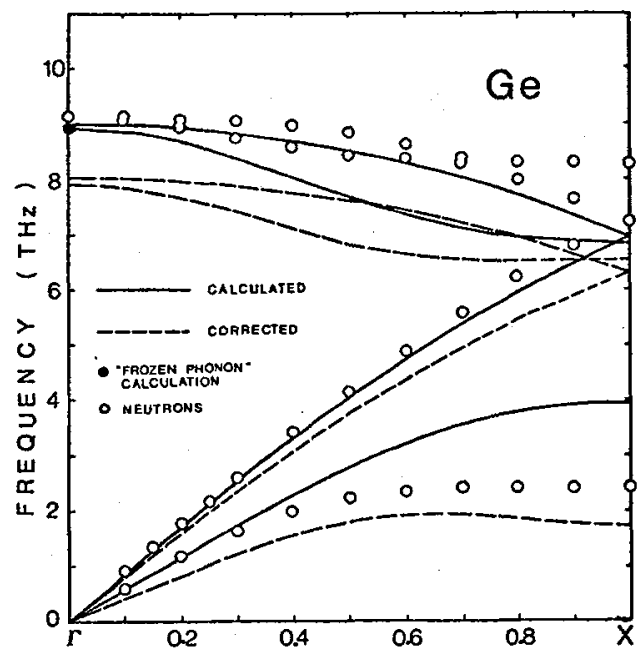

Fig. 2: Phonon dispersion along [100] predicted from forces acting on 8 atoms of the periodic supercell in the displacement patterns of Fig. la,b (solid line). The LO and TO branches - although provided by two different and completely independent calculations converge to the same frequency at $\boldsymbol{\Gamma}$ to within $1.1 \%$. This limit is consistent with the energy of the degenerate LTO( $\Gamma)$ mode determined by the alternative "frozen phonon" approach, in the framework of the same local charge-density functional and using the same pseudopotential. The ionic potential used for determination of electronic charge-densities predicts the bonds by $8 \%$ too short, therefore we have added approximate corrections for the contribution caused by the inadequacy of the potential (broken lines). Experimental points taken from Ref. 7 correspond to $80 \mathrm{~K}$.

Presently we can eliminate the anharmonicity because, after repeating the transverse calculations with displacement $u=-0.005 a$, we obtained the same force constants to within less than $1 \%$. We believe (3) and (4) to be the principal causes of the remaining discrepancies: Our method of correcting assigned the spurious contribution to only the first neighbor interactions which is likely to be a simplification of reality, and which is merely the least artificial among all possible assumptions. On the other hand, by performing calculations on quadrupled unit cell (rather than sextupled or octupled) we have limited the range of interactions to 4 th neighbors while Herman [6], on the basis of the neutron data, has shown that inclusion of the 5th neighbors is essential for the lattice dynamics of Ge. Calculations on larger cells to determine longer range force constants are in progress.

\section{References}

[1] P. Hohenberg and W. Kohn, Phys. Rev. 136, B864 (1964); W. Kohn and L. J. Sham, ibid. 140, A1133 (1965).

[2] H. Wendel and R. M. Martin, Phys. Rev. B 19, 5251 (1979); M. T. Yin and M. L. Cohen, Phys. Rev. Lett. 45, 1004 (1980); B. N. Harmon, W. Weber, D. R. Hamann, to be published.

[3] K. Kunc and R. M. Martin, Phys. Rev. B (to be published, August 15th, 1981).

[4] W. E. Pickett, S. G. Louie and M. L. Cohen, Phys. Rev. B 17, 815 (1978).

[5] K. Kunc and R. M. Martin, Phys. Rev. B (to be published, Sept 15th, 1981).

[6] F. Herman, J. Phys. Chem. Solids 8, 405 (1959).

[7] G. Nilsson and G. Nelin, Phys. Rev. B 3, 364 (1971). 